\title{
Irradiation pretreatment enhances the therapeutic efficacy of platelet-membrane-camouflaged antitumor nanoparticles
}

\author{
Yin Chen ${ }^{1 \dagger}$, Xue Shen ${ }^{2 \dagger}$, Songling Han ${ }^{1}$, Tao Wang ${ }^{1}$, Jianqi Zhao ${ }^{1}$, Yongwu He ${ }^{1,3}$, Shilei Chen ${ }^{1}$, Shengqi Deng ${ }^{2}$,
} Cheng Wang $^{1 *}$ (D) and Junping Wang ${ }^{1 *}$

\begin{abstract}
Background: Cell membrane-based nanocarriers are promising candidates for delivering antitumor agents. The employment of a simple and feasible method to improve the tumor-targeting abilities of these systems is appealing for further application. Herein, we prepared a platelet membrane (PM)-camouflaged antitumor nanoparticle. The effects of irradiation pretreatment on tumor targeting of the nanomaterial and on its antitumor action were evaluated.
\end{abstract}

Results: The biomimetic nanomaterial constructed by indocyanine green, poly(d,l-lactide-co-glycolide), and PM is termed PINPs@PM. A 4-Gy X-ray irradiation increased the proportions of G2/M phase and Caveolin-1 content in 4T1 breast cancer cells, contributing to an endocytic enhancement of PINPs@PM.PINPs@PM produced hyperthermia and reactive oxygen species upon excitation by near-infrared irradiation, which were detrimental to the cytoplasmic lysosome and resulted in cell death. Irradiation pretreatment thus strengthened the antitumor activity of PINPs@PM in vitro. Mice experiments revealed that irradiation enhanced the tumor targeting capability of PINPs@PM in vivo. When the same dose of PINPs@PM was intravenously administered, irradiated mice had a better outcome than did mice without X-ray pretreatment.

Conclusion: The study demonstrates an effective strategy combining irradiation pretreatment and PM camouflage to deliver antitumor nanoparticles, which may be instrumental for targeted tumor therapy.

\section{Background}

Cancer is a global threat to human health [1]. Surgery, radiotherapy, and chemotherapy are conventional methods to treat cancers, but they all have inherent limitations in clinical applications, such as invasiveness, drug

\footnotetext{
*Correspondence: wangctmmu@126.com; wangjunping@tmmu.edu.cn

†Yin Chen and Xue Shen contributed equally to this work

1 State Key Laboratory of Trauma, Burns and Combined Injury, Institute of Combined Injury of PLA, Chongqing Engineering Research Center for Nanomedicine, College of Preventive Medicine, Third Military Medical University, Chongqing 400038, China

Full list of author information is available at the end of the article
}

resistance, and severe side effects [2]. Phototherapy, including photothermal therapy (PTT) and photodynamic therapy (PDT), is a noninvasive and effective antineoplastic strategy and is considered a promising alternative to classical oncotherapy [3]. PTT and PDT eliminate cancer cells based on the fact that after excitation with light of a specific wavelength, photothermal agents and photosensitizers generate hyperthermia and reactive oxygen species (ROS), respectively, which are detrimental to cancer cells $[4,5]$.

Indocyanine green (ICG), one of the near-infrared dyes approved by the US Food and Drug Administration for 
clinical imaging and diagnosis, is a photothermal agent as well as a photosensitizer and thus attracts considerable attention. Because ICG lacks a tumor-targeting ability and tends to be rapidly cleared in vivo, many nanocarriers have been developed to deliver ICG [6]. Nevertheless, provided that the carrier is not endowed with antiphagocytic ability against the mononuclear phagocyte system in vivo, the bioavailability of ICG is still limited [7]. Cell membrane-based nanoparticles (CMBNPs) represent promising materials to overcome this shortcoming [8], as the functional molecules on the membrane, such as CD47 [9], CD45, and glycans, can send a "don't eat me" signal to the immune system [10]. The biomimetic strategy is plausibly beneficial for ICG delivery.

Intensifying the tumor-targeting ability of nanocarriers is also instrumental for ICG delivery. Passive and active targeting strategies are employed by therapeutic nanoparticles (TNPs) to reach the tumor site. Compared with the passive targeting phenomenon, which is mostly based on the enhanced permeability and retention effect and is limited by tumor types [11, 12], the active targeting method, such as modifying TNPs with peptides and antibodies [13, 14], is more efficient to promote drug accumulation in tumors. Notably, due to the presence of functional molecules with high affinity to cancer cells on certain cell membranes, P-selectin on the platelet membrane (PM) [4], for example, some CMBNPs possess an active tumor targeting capability. Further decoration of the cell membrane with tumor necrosis factor-related apoptosis-inducing ligand can strengthen the targeting ability [15], but the process is somewhat complex. The employment of simple and feasible methods that can help to target these CMBNPs to the tumor site is appealing for future clinical use.

In this study, we employed a PM-camouflaged poly(d,l-lactide-co-glycolide) (PLGA) nanocarrier to deliver ICG, obtaining a composite termed PINPs@PM (Fig. 1). The effect of X-ray irradiation on the antitumor activity of PINPs@PM in the presence of near-infrared irradiation (NIR) in vitro and in vivo was evaluated. The combined strategy using irradiation pretreatment and cell membrane camouflage is efficient for delivering drugs to tumors and thus has wide prospects in tumor diagnosis and therapy.

\section{Results and discussion}

\section{Preparation and characterization of PINPs@PM}

The ICG-loaded PLGA nanoparticles fabricated using a double emulsion method were termed PINPs (Fig. 1). The encapsulation efficiency of ICG was $60.6 \%$, calculated by ultraviolet spectrophotometry as we recently described [16]. PINPs were spherical with a good dispersion ability (Additional file 1). After decoration with a PM, the nanoparticles became PINPs@PM which exhibited white halos by transmission electron microscopy (TEM, Fig. 2a). Sodium dodecyl sulfate-polyacrylamide gel electrophoresis (SDS-PAGE) confirmed the successful coating of PM (Fig. 2b) [17]. BCA assay indicated that the average coating efficiency of PM on PINPs was $57.3 \%$. The zeta potential determination revealed that the PM camouflage increased the surface charge of the PINPs (averagely $-31 \mathrm{mV}$ ) to approximately $-20 \mathrm{mV}$, which was similar to the charge of free platelets (Fig. 2c). The hydrodynamic diameter of the PINPs@PM (Size=255 nm, PDI =0.143) measured by dynamic laser scattering (DLS) was slightly larger than that of the PINPs (Fig. 2d, Size $=219 \mathrm{~nm}$, PDI $=0.118$ ), which was consistent with the influence of red blood cell membrane cloaking on PLGA nanoparticles encapsulating perfluorocarbons [18].

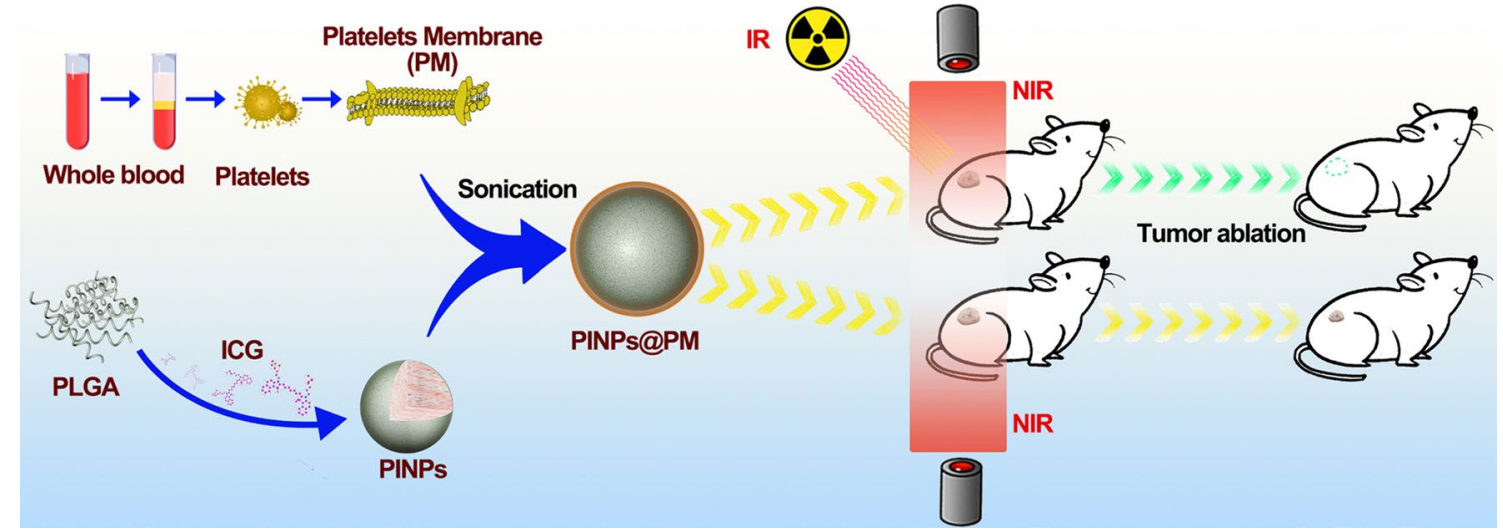

Fig. 1 Diagrammatic drawing depicting the preparation and evaluation of PINPs@PM 


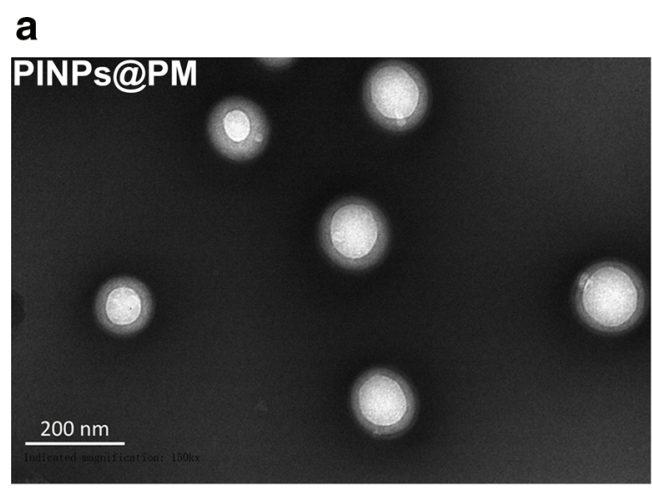

b
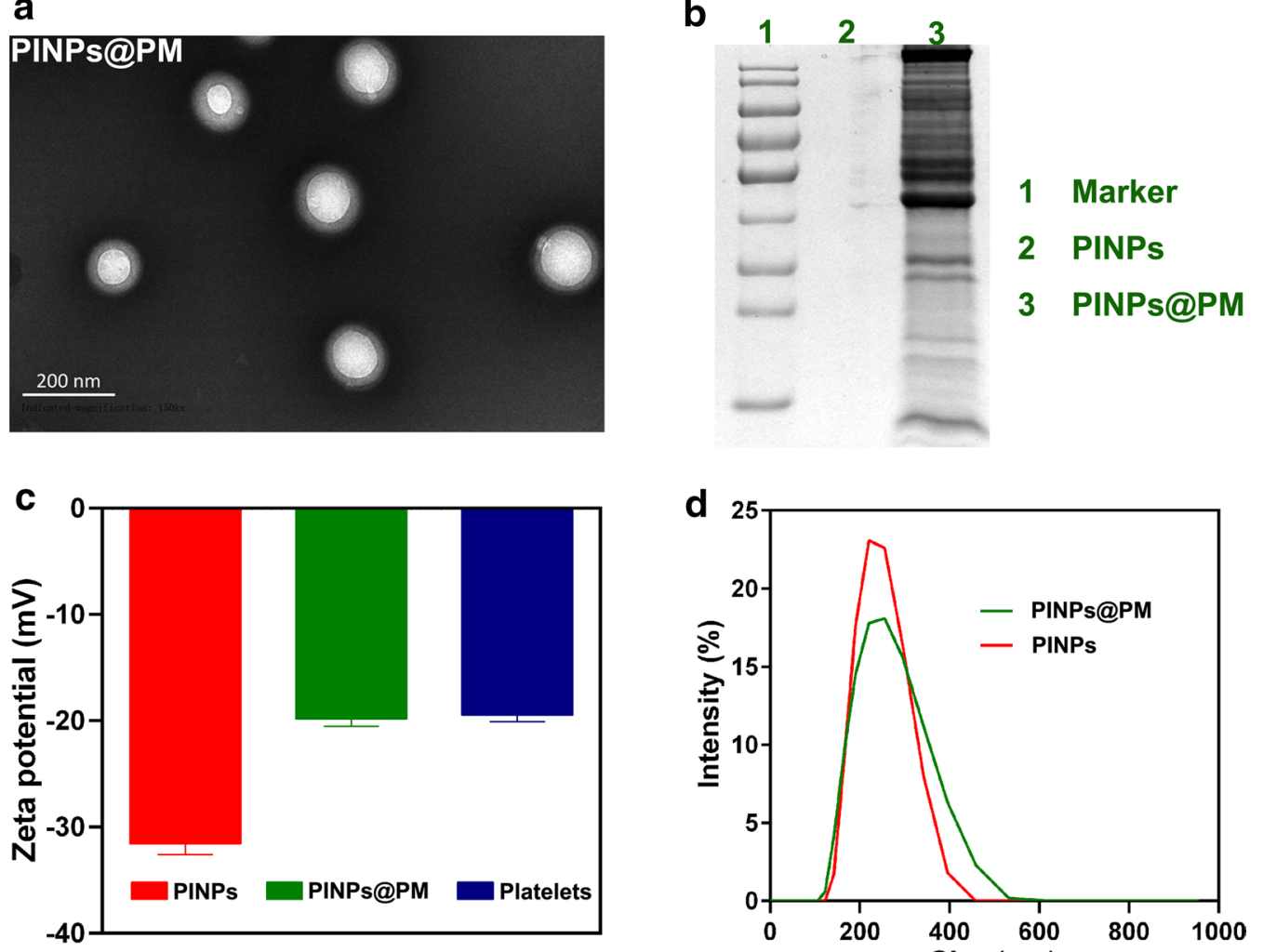

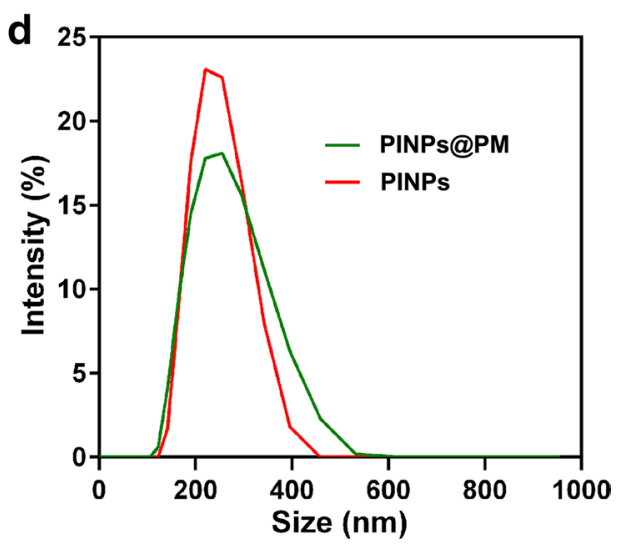

Fig. 2 Characterization of PINPs@PM. a TEM image of PINPs@PM. The scale is 200 nm. b SDS-PAGE analysis. The PM protein (20 $\mu \mathrm{g})$ was resolved by $10 \%$ SDS-PAGE. c Surface charge determination. The results are shown as the mean \pm SD. $\mathbf{d}$ DLS detection of the hydrodynamic diameters

\section{In vitro and in vivo toxicological evaluation}

To assess the material toxicity, we incubated PINPs@PM with human umbilical vein endothelial cell (HUVEC) and mouse 4T1 cells for 24 h. PINPs@PM had less of an effect on the cell survival at concentrations up to $50 \mu \mathrm{g} /$ $\mathrm{ml}$ (based on the concentration of ICG, Fig. 3a). The marginal haemolytic property of PINPs@PM further confirmed its biosafety in vitro (Fig. 3b). Animal experiments showed that intravenous injection of $60 \mu \mathrm{g}$ of PINPs@PM $(300 \mu \mathrm{g} / \mathrm{ml}$, based on the dose of ICG) was nonlethal to $\mathrm{BALB} / \mathrm{c}$ mice. The vital organs including the heart, liver, spleen, lung, and kidney were obtained after 3 weeks. In line with the nontoxicity of CMBNPs [19], there were no pathological changes in the tissue revealed by haematoxylin and eosin (HE) staining (Fig. 3c), indicative of the biocompatibility of PINPs@PM in vivo.

\section{Effect of irradiation on the endocytosis of PINPs@PM by cancer cells}

Platelets have pathophysiological affnity with tumors [20]. The interaction between P-selectin and CD44 partially contributes to the binding of PM-camouflaged nanomaterials to the surface of breast cancer cells $[4,21]$.
After aggregation on the cell membrane, our data showed that PINPs@PM was endocytosed by 4T1 cells in a timedependent manner. Notably, pretreatment of the cells with a 4-Gy X-ray irradiation $(1.0 \mathrm{~Gy} / \mathrm{min})$ enhanced the endocytosis without inducing severe membrane damage (Additional file 2). Flow cytometry revealed that the fluorescence intensity of $4 \mathrm{~T} 1$ cells phagocytosing $30 \mu \mathrm{g} / \mathrm{ml}$ PINPs@PM after $12 \mathrm{~h}$ averaged 12,332, which was significantly higher than that of cells without irradiation (average of 10,696, Fig. 4a). We measured the cell cycle of 4T1 cells $12 \mathrm{~h}$ post irradiation and found that the proportions of $\mathrm{G} 2 / \mathrm{M}$ phase and G0/G1 phase cells were increased from $15.43 \%$ to $65.63 \%$ and decreased from 22.6 to $6.85 \%$, respectively (Fig. 4b). Additionally, Caveolin-1, a membrane protein to constitute caveolae [22], was increased post irradiation (Fig. 4c). Because cells in the G2/M and G0/G1 phases are the most efficient and inefficient to uptake nanoparticles [23], and because over-expression of Caveolin-1 benefits material endocytosis by cancer cells [24], the alterations in cell cycle and Caveolin-1 content we considered contributed to the enhancement effect of X-ray irradiation on the uptake of PINPs@PM by $4 \mathrm{~T} 1$ cells. 

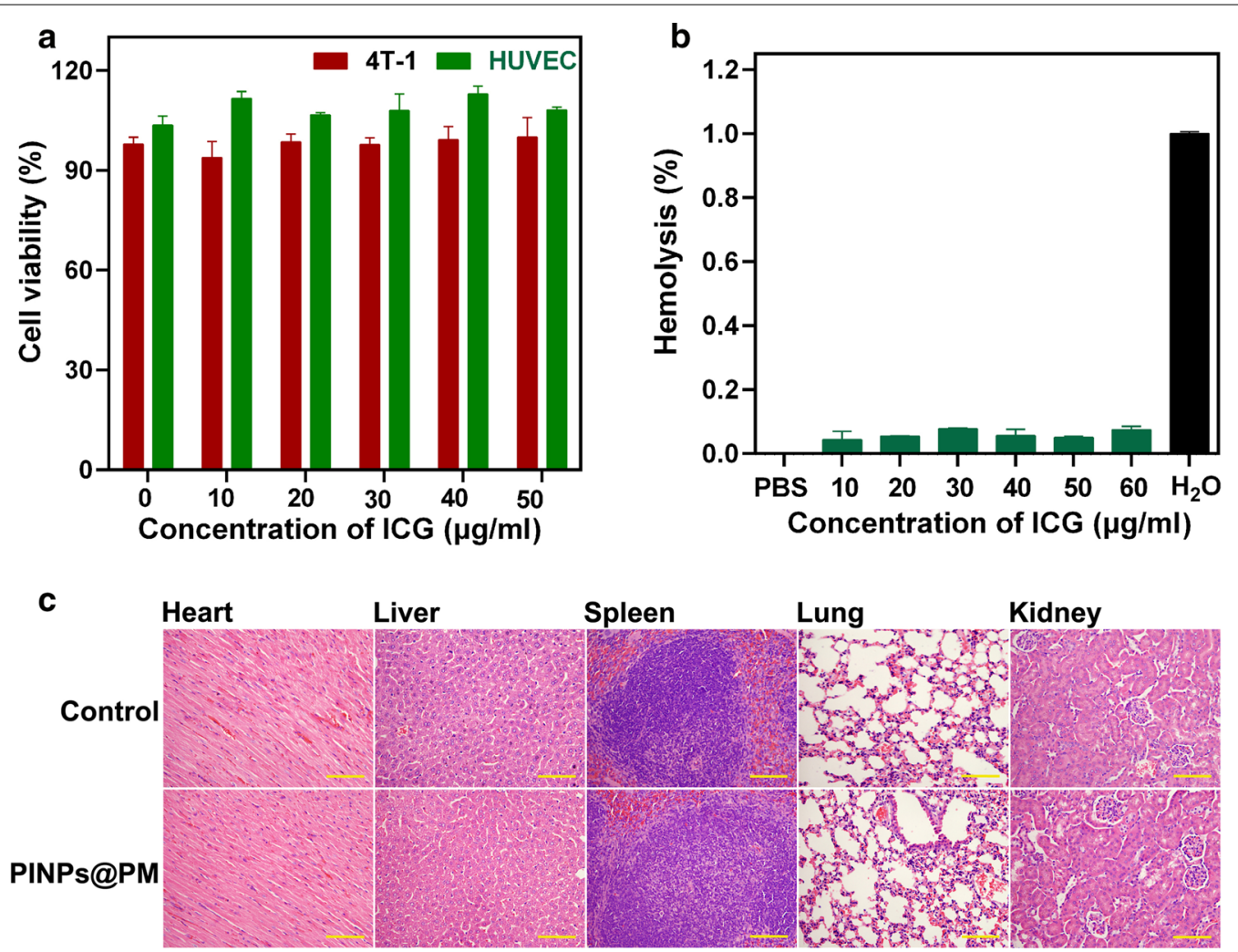

Fig.3 Toxicity evaluation of PINPs@PM. a The survival of HUVECs and 4T1 cells exposed to different concentrations of PINPs@PM for 24 h. The results are shown as the mean \pm SD. $\mathbf{b}$ Haemolysis of different concentrations of PINPs@PM. c HE staining of the organs of mice treated with NaCl and PINPs@PM. The scale is $100 \mu \mathrm{m}$

\section{Antitumor evaluation in vitro}

ICG is a photosensitizer and a photothermal agent. After the endocytosis by 4T1 cells, PINPs@PM produced intracellular ROS that could be stained by $2^{\prime}, 7^{\prime}$-dichlorodihydrofluorescein diacetate (DCFH-DA) probes upon excitation by NIR at $808 \mathrm{~nm}$ (Fig. 5a). The green fluorescence of X-ray-treated cells was visually stronger than that of cells without irradiation, which also indirectly indicated that irradiation pretreatment could strengthen the endocytosis of PINPs@PM by cancer cells. ROS are closely related to the growth and death of cancer cells [25]. On the one hand, ROS can induce the DNA mutation and genomic instability, accelerating the proliferation, immune tolerance, and metastasis of cancer cells $[26,27]$. In addition, high ROS levels enhance the cellular oxidative stress, which is detrimental to DNA, proteins, and lipids and thus causes cell death [28]. Ample studies have exploited the modulation of oxidative stress by delivering photosensitizers to the tumor site as the therapeutic strategy [29-31].

Otherwise, the temperature of PINPs@PM increased with increasing exposure time to NIR (Fig. 5b). After $10 \mathrm{~min}$ of NIR $\left(808 \mathrm{~nm}, 1 \mathrm{~W} / \mathrm{cm}^{2}\right)$, the temperature of $30 \mu \mathrm{g} / \mathrm{ml}$ PINPs@PM reached $56.8{ }^{\circ} \mathrm{C}$, which is sufficient to damage proteins and inactivate cancer cells [32]. Accordingly, with the actions of excess ROS and hyperthermia, the $4 \mathrm{~T} 1$ cells were eliminated in a dosedependent manner by PINPs@PM (Fig. 5c). Similar to the endocytic tendency shown in Fig. 4a, X-ray irradiation pretreatment significantly intensified the antitumor activity of PINPs@PM in vitro.

Lysosomes are considered therapeutic targets to treat many diseases including rheumatoid arthritis, Alzheimer's disease, and cancers [33, 34]. Lysosome is the intracellular target of some nanomaterials and its permeabilization is involved in material-mediated cell death [35]. Inhibiting lysosome function enhances the antitumor activity of chemotherapeutic drugs and increases the sensitivity of tumors to radiotherapy [36, 37]. We employed the acridine orange (AO) dye to stain 4T1 cells. Normally, the intact acidic lysosome exhibits red fluorescence, while the cytosol and nuclei exhibit green fluorescence upon excitation at $488 \mathrm{~nm}$. Fluorescence microscopy showed that the red fluorescence in cells treated with X-ray/PINPs@PM/NIR was dramatically decreased compared with that in PBS-treated cells 


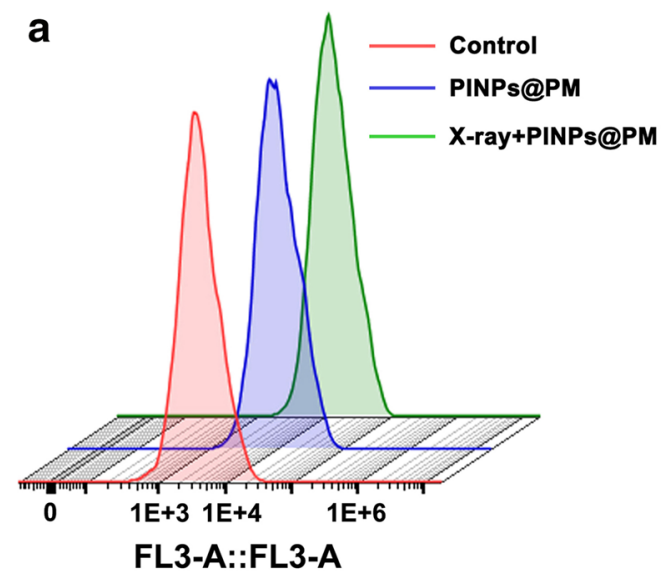

b

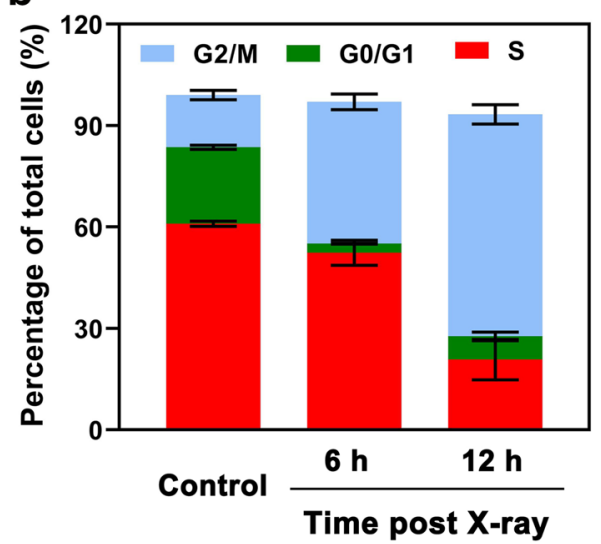

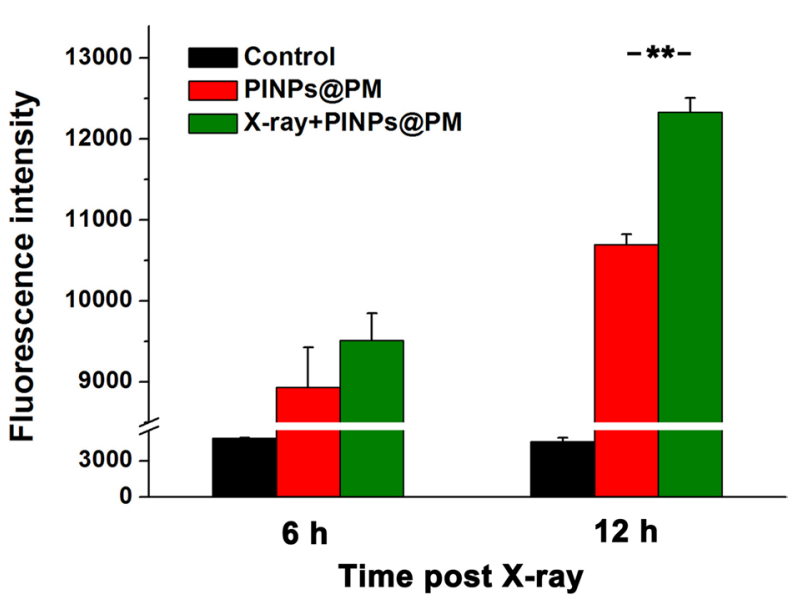

C

\section{Caveolin-1}

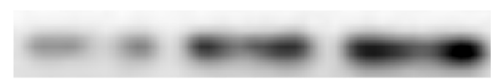

B-Actin

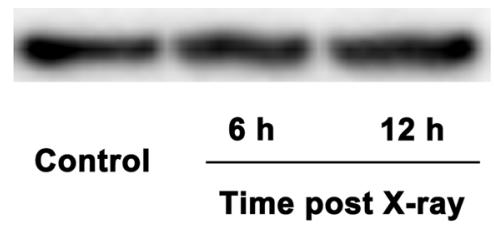

Fig. 4 Cellular uptake of PINPs@PM post irradiation. a Flow cytometry of 4T1 cells treated with PINPs@PM for $12 \mathrm{~h}$ in the presence and absence of irradiation pretreatment. The fluorescence intensity is presented as the mean \pm SD. ${ }^{* *}, P<0.01$. $\mathbf{b}$ Cell cycle distribution of 4T1 cells treated with $4-\mathrm{Gy}$ $X$-ray irradiation. The proportions of each phase are presented as the mean \pm SD. $\mathbf{c}$ Immunoblotting revealed the alterations of Caveolin-1 in 4T1 cells after irradiation. $\beta$-actin was employed as the reference

(Fig. 5d), suggesting that the lysosome is severely injured by the combined treatment. Due to the photochemical internalization effect of ROS on the membrane of lysosomes and the photothermal effect of ICG, the ROS and hyperthermia generated by NIR-treated PINPs@PM may be responsible for the lysosome disruption [38], which partially contributes to cell death.

\section{Tumor targeting and therapeutic assessment in vivo}

We obtained the fluorescent images of 4T1 tumorbearing mice intravenously administered with $60 \mu \mathrm{g}$ of PINPs@PM 12 h (Additional file 3) and 24 h (Fig. 6a) post irradiation. Because irradiation not only enhances the material endocytosis by cancer cells but also enables the alteration of the tumor microenvironment to accumulate nanoparticles [39], PINPs@PM accumulated more at the tumor site with the assistance of X-ray than did the group without irradiation pretreatment, which was also evidenced by the in vitro tissue imaging (Fig. 6b). The infrared thermal imaging supported that the maximal tumor temperature of PINPs@PM-treated mice reached $41.2^{\circ} \mathrm{C}$ after $10 \mathrm{~min}$ of NIR treatment (Fig. 6c), which was much higher than that of $\mathrm{NaCl}$-treated mice $\left(30.3^{\circ} \mathrm{C}\right.$, Additional file 4) but lower than that of X-ray-pretreated mice $\left(46.9^{\circ} \mathrm{C}\right)$. In contrast to the PINPs@PM/NIR group whose temperature tended to balance after $8 \mathrm{~min}$, the X-ray/PINPs@PM/NIR group exerted an overt timedependent temperature increase (Fig. 6d).

After NIR treatment, mice were monitored for 21 days and sacrificed, and the tumors were obtained by surgery (Fig. 7a). Consistent with that PM cloaking improves the therapeutic efficacy of mesoporous silica-coated bismuth nanorod as we recently described [4], PINPs@PM was superior to PINPs at decreasing the tumor volumes (Additional file 5). Due to the enhancement to tumor targeting, irradiation pretreatment further improved 

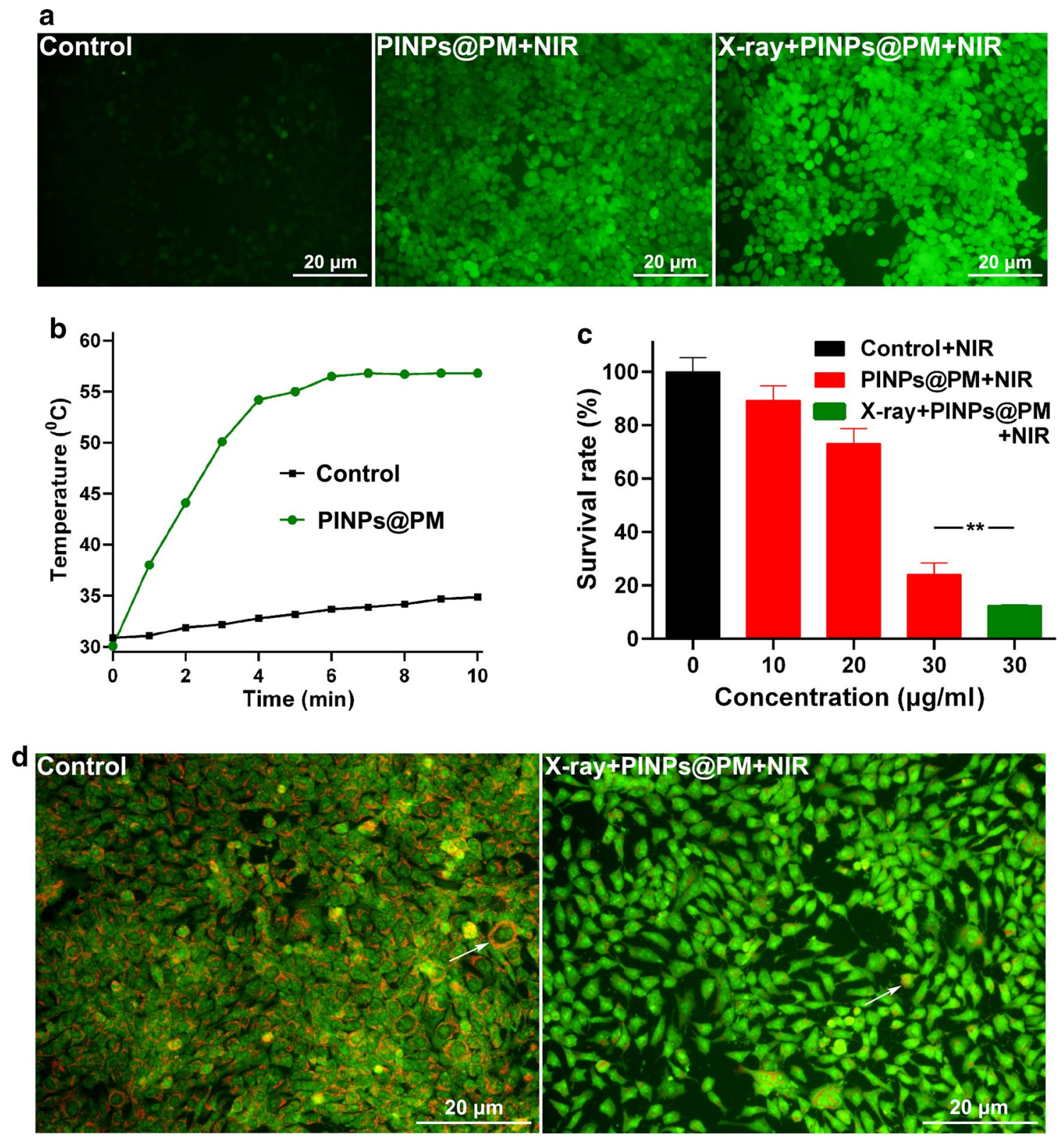

Fig.5 Irradiation pretreatment strengthens the antitumor activity of PINPs@PM in vitro. a ROS staining of 4T1 cells phagocytosing PINPs@PM in the presence and absence of X-ray irradiation. The scale is $20 \mu \mathrm{m}$. $\mathbf{b}$ The temperature of PINPs@PM $(30 \mu \mathrm{g} / \mathrm{ml})$ upon excitation by NIR for different times. c The survival of 4T1 cells treated with PINPs@PM in the presence and absence of irradiation pretreatment. ${ }^{* *}, P<0.01$. $\mathbf{d}$ AO staining showing the damage of lysosome in 4T1 cells. White arrows indicate the representative cytoplasmic lysosome. The scale is $20 \mu \mathrm{m}$

the curative effect of PINPs@PM, as revealed by the significantly lowered tumor sizes in both volume (Fig. 7b) and mass (Additional file 6). Terminal deoxynucleotidyl transferase-mediated deoxyuridinetriphosphate nick end labeling (TUNEL) assay showed that the dead cells in X-ray/PINPs@PM/NIR-treated tumors are visibly more than those in tumors without irradiation pretreatment (Fig. 7c). HE staining indicated that the structure of tumor tissue was severely destroyed after X-ray/PINPs@ $\mathrm{PM} / \mathrm{NIR}$ treatment.

\section{Conclusions}

ICG-based phototherapy is an effective strategy to treat tumors, whereas the circulation time of free ICG is short. In this study, we prepared a PM-camouflaged PLGA nanoparticle to deliver ICG, considering that 


\section{a}

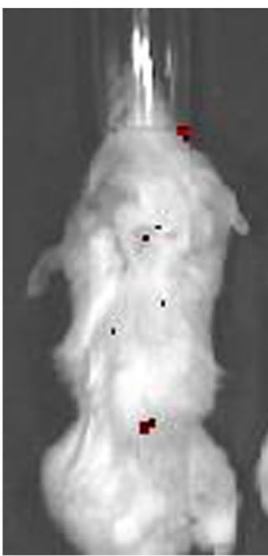

Control

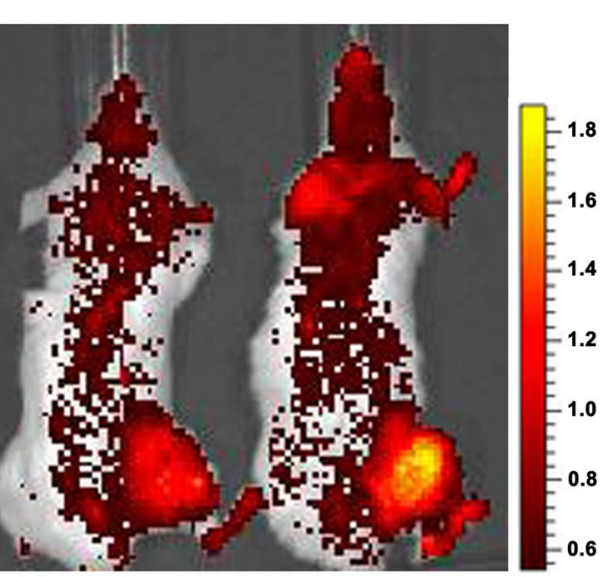

PINPs@PMX-ray+PINPs@PM b

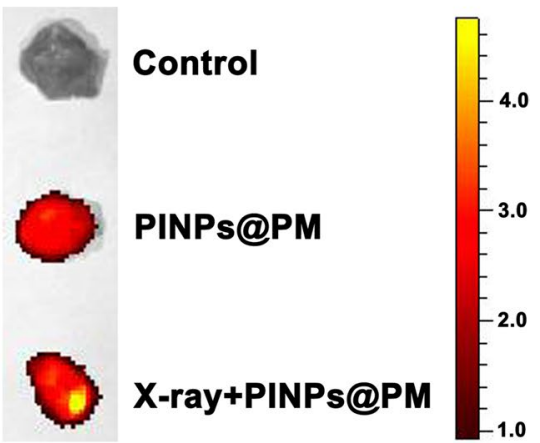

c

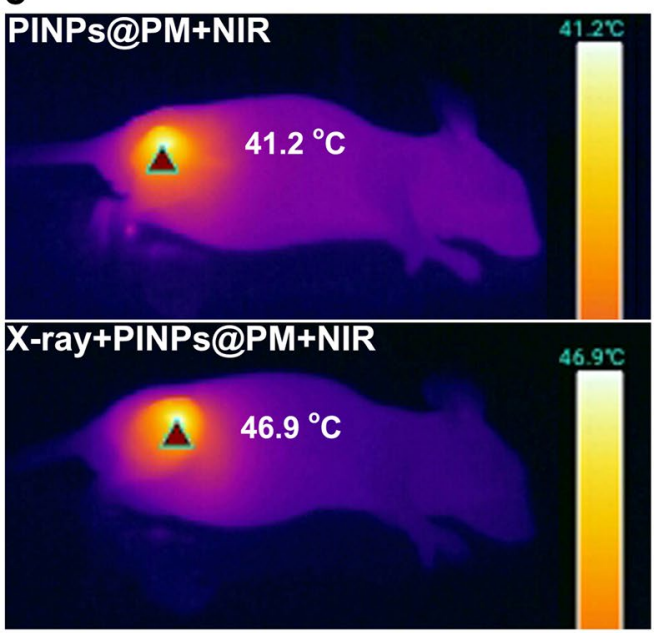

d

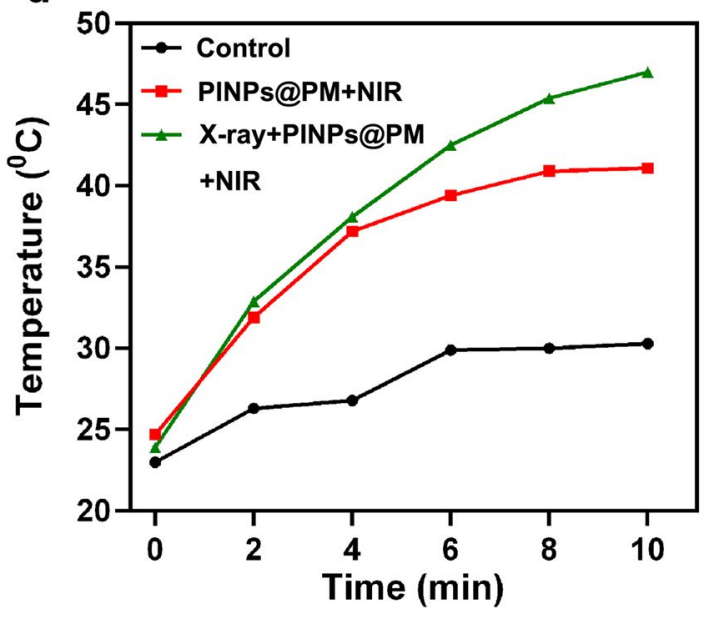

Fig. 6 Irradiation pretreatment enhances the accumulation of PINPs@PM in the tumor site. a In vivo imaging of tumor-bearing mice at $24 \mathrm{~h}$ post injection of PINPs@PM. b In vitro imaging of PINPs@PM in tumors. c Infrared thermal images of tumor-bearing mice treated with PINPs@PM in the presence and absence of X-ray irradiation.d The maximal tumor temperature of PINPs@PM-treated mice after NIR treatment for different times

PM camouflage not only lowers the immune clearance of nanocarriers but strengthens their tumor targeting capabilities. For further targeting enhancement, we optimized the therapeutic regimen by adding an irradiation pretreatment. The X-ray irradiation increased endocytosis of the nanomaterial by cancer cells and enhanced its targeting efficiency, thus intensifying its antitumor action in vitro and in vivo. The study demonstrating a combined use of irradiation pretreatment and PM camouflage to improve the targeting ability of antitumor nanoparticles is useful for further tumor therapy.

\section{Methods}

\section{Materials}

PLGA (50:50, inherent viscosity $0.20 \mathrm{dl} / \mathrm{g}, \mathrm{MW}=15,000$ ) was obtained from the Shandong Institute of Medical Instruments (Jinan, Shandong Province, CHN). Poly(vinyl alcohol) (PVA, MW=30,000-70,000, 78-90\% hydrolysed) and ICG were purchased from Sigma-Aldrich (St Louis, MO, US). RPMI 1640 cell culture medium and foetal bovine serum (FBS) were obtained from Gibco (Thermo Fisher Scientific, Shanghai, CHN). The SDSPAGE, DCFH-DA, and CCK-8 kits were obtained from Beyotime (Shanghai, CHN). The formaldehyde solution 
a

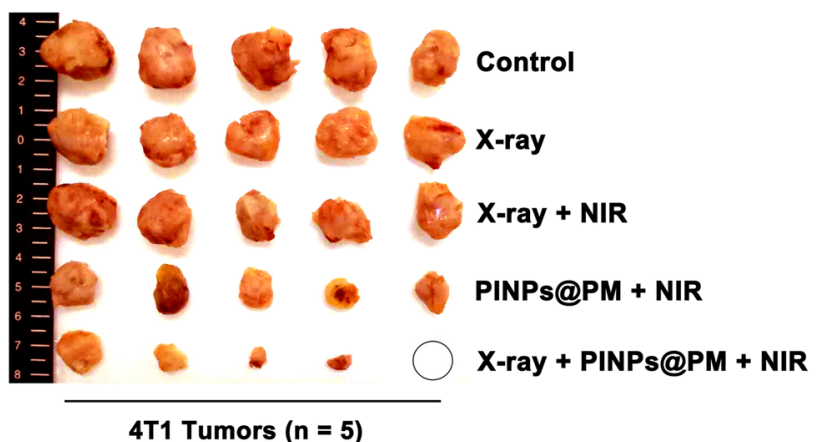

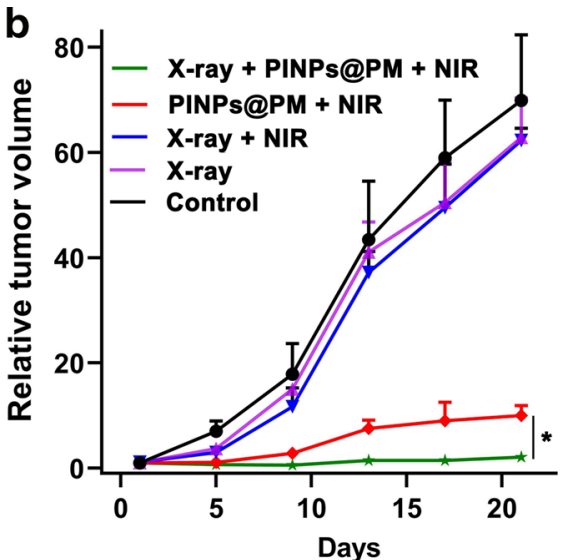

C

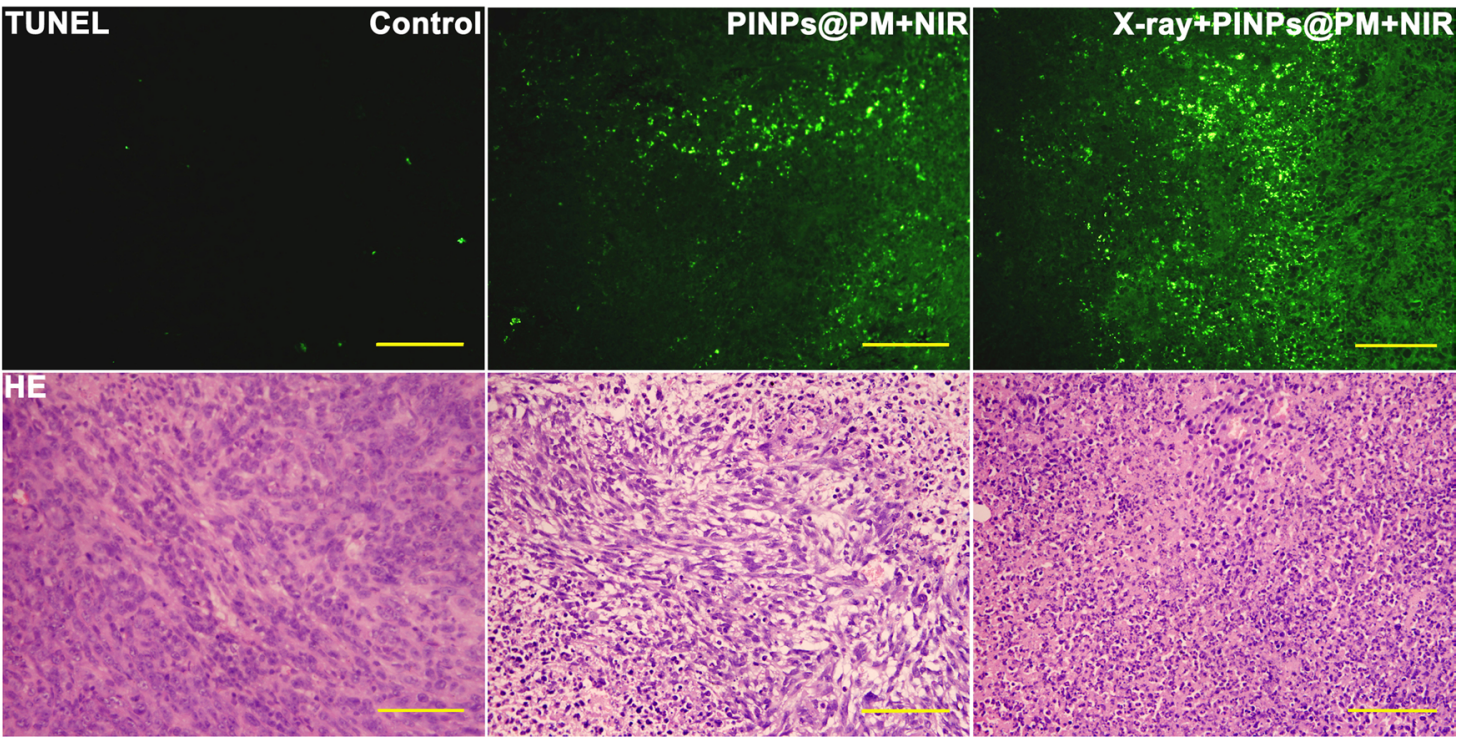

Fig. 7 Irradiation pretreatment improves the antitumor ability of PINPs@PM in vivo. a Photograph of mice tumors ( $n=5$ ). The circle indicates that the tumor is visually eliminated. $\mathbf{b}$ Dynamic alterations of tumor volumes. ${ }^{*}, P<0.05$. c TUNEL and HE staining showing the pathological changes of tumor tissues. The scale is $100 \mu \mathrm{m}$

(4\%), HE staining solution, and $\mathrm{AO}$ were purchased from Solarbio (Beijing, CHN). Deionized water was obtained from a Milli-Q water purification system.

\section{Preparation of PINPs and PINPs@PM}

The ICG-loaded PLGA nanoparticles (PINPs) were prepared using a reported water-in-oil-in-water double emulsion method with modifications [16, 40]. Briefly, PLGA $(120 \mathrm{mg}$ ) was dissolved in $2.5 \mathrm{ml}$ of methylene chloride. ICG solution $(7.5 \mathrm{mg} / \mathrm{ml}, 200 \mu \mathrm{l})$ was added, and the mixture was emulsified by sonication (25\% amplitude, 2 min) using a Digital Sonifier S-250D (Branson Ultrasonic, CT, US) in an ice bath. The primary emulsion (first emulsion) was poured into $10 \mathrm{ml}$ of PVA solution
$(3 \%, \mathrm{w} / \mathrm{v})$ and sonicated for another $3 \mathrm{~min}$, obtaining a double emulsion (second emulsion). The obtained product was stirred to evaporate the organic solvent and was then centrifuged at $1200 \mathrm{rpm}$ for $12 \mathrm{~min}$ to remove the non-encapsulated ICG.

PM camouflage was performed as we recently described [4]. Mouse platelets were collected by gradient centrifugation, frozen at $-80^{\circ} \mathrm{C}$ and thawed at room temperature. After three freeze-thaw cycles, the membrane was obtained by centrifugation at $8000 \mathrm{rpm}$ for $10 \mathrm{~min}$, washed with PBS containing protease inhibitor and sonicated for $5 \mathrm{~min}$. PINPs $(300 \mu \mathrm{g}$, based on the content of ICG) dissolved in PBS (1 ml) were added to equal volumes of PM. The mixture was then sonicated 
on ice for $2 \mathrm{~min}$ and maintained at $4{ }^{\circ} \mathrm{C}$ overnight. Large fragments of PM were removed using a filter membrane with a pore size of $400 \mathrm{~nm}$. The PINPs@PM was collected by high-speed centrifugation at 10,000 rpm for 15 min. A BCA assay was employed to determine the efficiency of membrane coating. Animals were cared for and treated in accordance with the National Institutes of Health (NIH) guidelines for the care and use of laboratory animals (NIH Publication No. 85e23 Rev. 1985) as approved by the Animal Experimental Ethics Committee of TMMU.

\section{Characterization analysis}

The morphologies of the PINPs and PINPs@PM were obtained by TEM (Tecnai G2 F20 U-TWIN, FEI, Hillsboro, OR, US). To confirm the PM camouflage, PINPs@ PM was denatured and resolved via 10\% SDS-PAGE. The protein bands were visualized by Coomassie blue staining. The DLS and zeta potential experiments were determined by a Nano-ZS (Malvern, Worcestershire, UK) at room temperature. The temperature alteration of PINPs@PM exposed to NIR at 808 nm for different times (1.0 W/ $\mathrm{cm}^{2}$, Laserwave, Beijing, $\mathrm{CHN}$ ) was recorded with a thermoelectric thermometer (HH806W, Omega, US).

\section{Toxicological evaluation}

HUVECs and 4T1 cells obtained from the Chinese Academy of Sciences (Shanghai, CHN) were cultured in RPMI 1640 medium containing 10\% FBS. Ten thousand cells cultured to the logarithmic phase were incubated with different concentrations of PINPs@PM $(0,10,20,30,40$, and $50 \mu \mathrm{g} / \mathrm{ml}$, based on the dose of ICG) for $24 \mathrm{~h}$. The cell viability was determined by CCK- 8 assay. Mouse erythrocytes diluted in saline solution $(2 \%, \mathrm{v} / \mathrm{v}, 300 \mu \mathrm{l})$ were incubated with $1.2 \mathrm{ml}$ of PINPs@PM at $37{ }^{\circ} \mathrm{C}$ for $2 \mathrm{~h}$. The absorbance of the supernatant was determined at $405 \mathrm{~nm}$. These experiments were conducted in triplicate and repeated twice. The toxicity of PINPs@PM in vivo was evaluated by a mouse experiment. Animals were cared for and treated as demonstrated in the preparation of PINPs@PM. PINPs@PM (60 $\mu$ g, based on the content of ICG) was intravenously injected. The mice were sacrificed after 7 days, and their hearts, livers, spleens, lungs, and kidneys were obtained by surgery. The pathological changes were observed with an Olympus DX51 optical microscope (Tokyo, JPN) after HE staining.

\section{Flow cytometry-based endocytic assay}

$4 \mathrm{~T} 1$ cells were seeded in a 6 -well plate at a density of $2 \times 10^{5}$ CFU per well and cultured overnight in RPMI

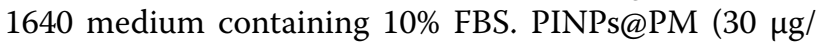
$\mathrm{ml}$, based on the content of ICG) was co-incubated with
$4 \mathrm{~T} 1$ cells for 6 and $12 \mathrm{~h}$ in the presence and absence of a 4-Gy X-ray irradiation pretreatment with an RS2000 $\mathrm{X}$-ray irradiator (1.0 Gy/min, Rad Source, Suwanee, GA). The counts of fluorescent cells uptaking ICG-loaded PINPs@PM excited at $780 \mathrm{~nm}$ and the fluorescence intensity were determined with a BD flow cytometry system (Franklin Lakes, NJ, US), in which 15,000 events per sample were obtained. The experiment was repeated three times, and the data were processed using FlowJo software (version 7.6.1).

\section{Cell cycle analysis}

$4 \mathrm{~T} 1$ cells $\left(2 \times 10^{5} \mathrm{CFU}\right)$ were cultured overnight in a 6-well plate and irradiated with a 4-Gy X-ray. After further incubation in RPMI 1640 medium containing 10\% FBS for 6 and $12 \mathrm{~h}$, cells were harvested by trypsinization and fixed with $70 \%$ ethanol at $4{ }^{\circ} \mathrm{C}$ for $24 \mathrm{~h}$. The cells were then stained with PI for $30 \mathrm{~min}$ after resuspension in RNase A buffer. The cell cycle was determined with a BD C6 flow cytometer. The data were processed using FlowJo software (version 7.6.1). This experiment was repeated three times on different days.

\section{Western blot}

4T1 cells irradiated with a 4-Gy X-ray and incubated for 6 and $12 \mathrm{~h}$ were collected and processed with RIPA lysis and extraction buffer $(89,900$, Thermo Fisher Scientific). The cell extracts were denatured and resolved via $10 \%$ SDS-PAGE. A primary anti-Caveolin-1 rabbit monoclonal antibody (Abcam, ab32577, 1:200, Shanghai, CHN) and a goat anti-rabbit secondary antibody (Abcam, ab205718, 1:1000) were employed to detect Caveolin-1. $\beta$-actin determined by a mouse monoclonal antibody (AA128, Beyotime, 1:1000) was used as a reference. This assay was repeated three times on different days.

\section{Antitumor cell experiment}

$4 \mathrm{~T} 1$ cells seeded in a 96-well plate at a density of $1 \times 10^{4} \mathrm{CFU}$ per well were cultured overnight and incubated with $30 \mu \mathrm{g} / \mathrm{ml}$ of PINPs@PM (based on the dose of ICG) in the presence and absence of a 4-Gy X-ray irradiation pretreatment. NIR $\left(808 \mathrm{~nm}, 1.0 \mathrm{~W} / \mathrm{cm}^{2}, 10 \mathrm{~min}\right)$ was performed after $12 \mathrm{~h}$ of co-incubation. Cells were then washed with sterile PBS, stained with $100 \mu \mathrm{l}$ of DCFH-DA $(10 \mu \mathrm{M})$ and AO dyes $(5 \mu \mathrm{g} / \mathrm{ml})$ according to the manufacturer's instructions and observed with an Olympus IX70 inverted fluorescence microscope. Otherwise, the cells were further incubated for $16 \mathrm{~h}$ followed by NIR treatment and the survival was detected by a CCK-8 assay. The experiment was conducted in duplicate and repeated three times. 


\section{Tumor targeting evaluation}

Female 8-week-old BALB/c mice were subcutaneously injected with $2 \times 10^{9} \mathrm{CFU} 4 \mathrm{~T} 1$ cells in the right hind leg. Animals were cared for and treated as demonstrated in the preparation of PINPs@PM. A 4-Gy X-ray local irradiation was performed when the tumor reached approximately 75 mm³.PINPs@PM (60 $\mu \mathrm{g}$, based on the content of ICG) was intravenously administered $6 \mathrm{~h}$ later. The accumulation of PINPs@PM in the tumor-bearing mice was observed with an IVIS spectrum in vivo imaging system (Perkin Elmer, Shanghai, CHN) at 12 and 24 h posttreatment. A Fortric $226 \mathrm{~s}$ thermal imager (Shanghai, $\mathrm{CHN}$ ) was used to obtain the infrared thermal images and record the temperature variations of the tumors along with the increasing NIR exposure times.

\section{Antitumor assay in vivo}

The tumor-bearing mice $(\mathrm{n}=5)$ were prepared and cared for as described in the tumor targeting evaluation. PINPs@PM (60 $\mu \mathrm{g}$, based on the content of ICG) was given by tail vein injection in the presence and absence of a 4-Gy X-ray local irradiation. NIR treatment was continued for $10 \mathrm{~min}$ after $24 \mathrm{~h}$. The tumor volumes were monitored for 3 weeks. The tumor tissues were obtained by surgery for weigh and TUNEL and HE staining.

\section{Statistical analysis}

The significant differences $(\mathrm{P})$ were calculated by SPSS 17.0 using the Student's two-tailed t test and LSD multiple-comparison test. A P value lower than 0.05 was defined as statistically significant.

\section{Supplementary information}

Supplementary information accompanies this paper at https://doi. org/10.1186/s12951-020-00660-z.

Additional file 1. TEM image of PINPS.

Additional file 2. 1-N-phenylnaph-thylamine (NPN)-uptake assay. Additional file 3. In vivo imaging of tumor-bearing mice at $12 \mathrm{~h}$ post injection of PINPs@PM.

Additional file 4. Thermal images of $\mathrm{NaCl}$-treated tumor-bearing mice. Additional file 5. PM camouflage improves the antitumor ability of PINPs in vivo.

Additional file 6. Histogram showing the tumor weight at day 21.

\section{Acknowledgements}

We appreciate Dr. Changhong Du (College of Preventive Medicine, Third Military Medical University) for the assistance in flow cytometry analysis.

\section{Authors' contributions}

CY and SX performed the experiments. WC, WJ designed the experiments and drafted the manuscript. HS and WT contributed to the data analysis. CS and DS contributed to the manuscript preparation and review. ZJ and
HY participated in the animal experiments. All authors approved the final manuscript.

\section{Funding}

This work was supported by Grants from the National Natural Science Fund of China (Nos. 81725019, 81930090, and 81703395), the Fund from PLA (No. AWS16J014), and the Natural Science Funds of Chongqing City (Nos. cstc2019jcyj-msxmX0011 and cstc2019jcyjjqX0005)

\section{Availability of data and materials}

All data generated or analyzed during this study are included in this published article.

\section{Ethics approval and consent to participate}

The study was approved by Ethics Committee of TMMU.

\section{Consent for publication}

All the authors have approved the manuscript and agree with submission to your esteemed journal.

\section{Competing interests}

The authors declare that they have no competing interests.

\section{Author details}

${ }^{1}$ State Key Laboratory of Trauma, Burns and Combined Injury, Institute of Combined Injury of PLA, Chongqing Engineering Research Center for Nanomedicine, College of Preventive Medicine, Third Military Medical University, Chongqing 400038, China. ${ }^{2}$ Sichuan Industrial Institute of Antibiotics, Chengdu University, Chengdu 610106, China. ${ }^{3}$ College of Materials Science and Engineering, Hebei University of Engineering, Handan 056038, China.

Received: 20 December 2019 Accepted: 13 July 2020

Published online: 20 July 2020

\section{References}

1. Torre LA, Bray F, Siegel RL, Ferlay J, Lortet-Tieulent J, Jemal A. Global cancer statistics, 2012. CA Cancer J Clin. 2015;65:87-108.

2. Siegel RL, Miller KD, Jemal A. Cancer statistics, 2016. CA Cancer J Clin. 2016;66:7-30.

3. Wan G, Chen B, Li L, Wang D, Shi S, Zhang T, et al. Nanoscaled red blood cells facilitate breast cancer treatment by combining photothermal/photodynamic therapy and chemotherapy. Biomaterials. 2018;155:25-40.

4. Chen Y, Zhao G, Wang S, He Y, Han S, Du C, et al. Platelet-membrane-camouflaged bismuth sulfide nanorods for synergistic radio-photothermal therapy against cancer. Biomater Sci. 2019;7:3450-9.

5. Yuan Z, Tao B, He Y, Mu C, Liu G, Zhang J, et al. Remote eradication of biofilm on titanium implant via near-infrared light triggered photothermal/ photodynamic therapy strategy. Biomaterials. 2019;223:119479.

6. Liu Y, Chen S, Sun J, Zhu S, Chen C, Xie W, et al. Folate-targeted and oxygen/indocyanine green-loaded lipid nanoparticles for dual-mode imaging and photo-sonodynamic/photothermal therapy of ovarian cancer in vitro and in vivo. Mol Pharm. 2019;17:1442-3.

7. Jing $L, Q u H$, Wu D, Zhu C, Yang Y, Jin X, et al. Platelet-camouflaged nanococktail: simultaneous inhibition of drug-resistant tumor growth and metastasis via a cancer cells and tumor vasculature dual-targeting strategy. Theranostics. 2018;8:2683-95.

8. Soprano E, Alvarez A, Pelaz B, Del Pino P, Polo E. Plasmonic cell-derived nanocomposites for light-controlled cargo release inside living cells. Adv Biosyst. 2020;4:e1900260.

9. Oldenborg P-A, Zheleznyak A, Fang Y-F, Lagenaur CF, Gresham HD, Lindberg FP. Role of CD47 as a marker of self on red blood cells. Science. 2000;288:2051-4.

10. Parodi A, Quattrocchi N, van de Ven AL, Chiappini C, Evangelopoulos M, Martinez JO, et al. Synthetic nanoparticles functionalized with biomimetic leukocyte membranes possess cell-like functions. Nat Nanotechnol. 2012;8:61.

11. Yang H, Chen Y, Chen Z, Geng Y, Xie X, Shen X, et al. Chemo-photodynamic combined gene therapy and dual-modal cancer imaging achieved 
by $\mathrm{pH}$-responsive alginate/chitosan multilayer-modified magnetic mesoporous silica nanocomposites. Biomater Sci. 2017;5:1001-13.

12. Prabhakar U, Maeda H, Jain RK, Sevick-Muraca EM, Zamboni W, Farokhzad $\mathrm{OC}$, et al. Challenges and key considerations of the enhanced permeability and retention effect for nanomedicine drug delivery in oncology. Cancer Res. 2013;73:2412-7.

13. Chai Z, Hu X, Wei X, Zhan C, Lu L, Jiang K, et al. A facile approach to functionalizing cell membrane-coated nanoparticles with neurotoxinderived peptide for brain-targeted drug delivery. J Control Release. 2017;264:102-11.

14. Li L, Lu Y, Jiang C, Zhu Y, Yang X, Hu X, et al. Actively targeted deep tissue imaging and photothermal-chemo therapy of breast cancer by antibody-functionalized drug-loaded X-ray-responsive bismuth sulfide@mesoporous silica core-shell nanoparticles. Adv Funct Mater. 2018;28:1704623.

15. Hu Q, Sun W, Qian C, Wang C, Bomba HN, Gu Z. Anticancer plateletmimicking nanovehicles. Adv Mater. 2015;27:7043-50.

16. Shen X, Li T, Chen Z, Xie X, Zhang H, Feng Y, et al. NIR-light-triggered anticancer strategy for dual-modality imaging-guided combination therapy via a bioinspired hybrid PLGA nanoplatform. Mol Pharm. 2019;16:1367-84.

17. Rao L, Bu L-L, Meng Q-F, Cai B, Deng W-W, Li A, et al. Antitumor plateletmimicking magnetic nanoparticles. Adv Funct Mater. 2017;27:1604774.

18. Gao M, Liang C, Song X, Chen Q, Jin Q, Wang C, et al. Erythrocyte-membrane-enveloped perfluorocarbon as nanoscale artificial red blood cells to relieve tumor hypoxia and enhance cancer radiotherapy. Adv Mater. 2017;29:1701429.

19. Li R, He Y, Zhang S, Qin J, Wang J. Cell membrane-based nanoparticles: a new biomimetic platform for tumor diagnosis and treatment. Acta Pharm Sin B. 2018:8:14-22.

20. Borsig $L$. The role of platelet activation in tumor metastasis. Expert Rev Anticancer Ther. 2008;8:1247-55.

21. Shang Y, Wang Q, Wu B, Zhao Q, Li J, Huang X, et al. Platelet-membranecamouflaged black phosphorus quantum dots enhance anticancer effect mediated by apoptosis and autophagy. ACS Appl Mater Interfaces. 2019;11:28254-66.

22. Dixit S, Sahu R, Verma R, Duncan S, Giambartolomei GH, Singh SR, et al. Caveolin-mediated endocytosis of the Chlamydia M278 outer membrane peptide encapsulated in poly(lactic acid)-Poly(ethylene glycol) nanoparticles by mouse primary dendritic cells enhances specific immune effectors mediated by MHC class II and CD4(+) T cells. Biomaterials. 2018;159:130-45.

23. Liu Y, Chen W, Zhang P, Jin X, Liu X, Li P, et al. Dynamically-enhanced retention of gold nanoclusters in HeLa cells following $X$-rays exposure: a cell cycle phase-dependent targeting approach. Radiother Oncol. 2016;119:544-51.

24. Ross NL, Sullivan MO. Overexpression of caveolin-1 in inflammatory breast cancer cells enables IBC-specific gene delivery and prodrug conversion using histone-targeted polyplexes. Biotechnol Bioeng. 2016;113:2686-97.
25. Sabharwal SS, Schumacker PT. Mitochondrial ROS in cancer: initiators, amplifiers or an Achilles'heel? Nat Rev Cancer. 2014;14:709.

26. Piskounova E, Agathocleous M, Murphy MM, Hu Z, Huddlestun SE, Zhao $Z$, et al. Oxidative stress inhibits distant metastasis by human melanoma cells. Nature. 2015;527:186.

27. Yu X, Lao Y, Teng X-L, Li S, Zhou Y, Wang F, et al. SENP3 maintains the stability and function of regulatory T cells via BACH2 deSUMOylation. Nat Commun. 2018;9:3157.

28. Gorrini C, Harris IS, MakTW. Modulation of oxidative stress as an anticancer strategy. Nat Rev Drug Discov. 2013;12:931.

29. Ren H, Liu J, Li Y, Wang H, Ge S, Yuan A, et al. Oxygen self-enriched nanoparticles functionalized with erythrocyte membranes for long circulation and enhanced phototherapy. Acta Biomater. 2017;59:269-82.

30. Wang H, Li X, Tse WC, Yang H, Thorling CA, Liu Y, et al. Indocyanine green-incorporating nanoparticles for cancer theranostics. Theranostics. 2018;8:1227-42.

31. Wang D, Niu L, Qiao Z-Y, Cheng D-B, Wang J, Zhong Y, et al. Synthesis of self-assembled porphyrin nanoparticle photosensitizers. ACS Nano. 2018;12:3796-803.

32. Kaur P, Hurwitz MD, Krishnan S, Asea A. Combined hyperthermia and radiotherapy for the treatment of cancer. Cancers (Basel). 2011;3:3799-823.

33. Bonam SR, Wang F, Muller S. Lysosomes as a therapeutic target. Nat Rev Drug Discov. 2019;18:923-48.

34. Mai TT, Hamai A, Hienzsch A, Caneque T, Muller S, Wicinski J, et al. Salinomycin kills cancer stem cells by sequestering iron in lysosomes. Nat Chem. 2017;9:1025-33.

35. Pérez-Hernández M, Del Pino P, Mitchell SG, Moros M, Stepien G, Pelaz B, et al. Dissecting the molecular mechanism of apoptosis during photothermal therapy using gold nanoprisms. ACS Nano. 2015;9:52-61.

36. Shao M, Zhu W, Lv X, Yang Q, Liu X, Xie Y, et al. Encapsulation of chloroquine and doxorubicin by MPEG-PLA to enhance anticancer effects by lysosomes inhibition in ovarian cancer. Int J Nanomed. 2018;13:8231-45.

37. Zhou W, Guo Y, Zhang $X$, Jiang Z. Lys05 induces lysosomal membrane permeabilization and increases radiosensitivity in glioblastoma. J Cell Biochem. 2020;121:2027-37.

38. Guo M, Mao H, Li Y, Zhu A, He H, Yang H, et al. Dual imaging-guided photothermal/photodynamic therapy using micelles. Biomaterials. 2014;35:4656-66

39. Miller MA, Chandra R, Cuccarese MF, Pfirschke C, Engblom C, Stapleton S, et al. Radiation therapy primes tumors for nanotherapeutic delivery via macrophage-mediated vascular bursts. Sci Transl Med. 2017;9:eaal0225.

40. Shen X, Li T, Chen Z, Geng Y, Xie X, Li S, et al. Luminescent/magnetic PLGA-based hybrid nanocomposites: a smart nanocarrier system for targeted codelivery and dual-modality imaging in cancer theranostics. Int J Nanomed. 2017;12:4299-322.

\section{Publisher's Note}

Springer Nature remains neutral with regard to jurisdictional claims in published maps and institutional affiliations.

\footnotetext{
Ready to submit your research? Choose BMC and benefit from:

- fast, convenient online submission

- thorough peer review by experienced researchers in your field

- rapid publication on acceptance

- support for research data, including large and complex data types

- gold Open Access which fosters wider collaboration and increased citations

- maximum visibility for your research: over $100 \mathrm{M}$ website views per year
}

At BMC, research is always in progress.

Learn more biomedcentral.com/submissions 